\title{
Impact of pesticide-fertilizer combinations on the rhizosphere microbiome of field-grown sugarcane
}

\author{
Weijuan Huang ${ }^{1}$, Yinglin $\mathrm{Lu}^{1}$, Lijun $\mathrm{Chen}^{1}$, and Yuxing $\mathrm{An}^{1}$ \\ ${ }^{1}$ Guangzhou Sugarcane Industry Research Institute
}

May 5, 2020

\begin{abstract}
The complex and enormous diversity of microbiome associated with plant roots is important for plant health and is shaped by numerous factors. This study aimed to unravel the effects of a pesticide-fertilizer combination on the rhizosphere microbiome of field-grown sugarcane. A field trial on sugarcane was conducted in Zhanjian city, Guangdong Province of China, and bulk soil and rhizosphere samples were collected 3 weeks after planting. We examined the effects of clothianidin and/or organic fertilizer treatments on the composition, diversity, and function of rhizosphere microbial communities using 16S rRNA gene and ITS1 gene amplicon sequencing. Compared with the controls (no pesticide or fertilizer used), the microbial community that resulted from treating with the pesticide-fertilizer (SPF) had a higher relative bacterial diversity and abundance, as well as contributing more comprehensive functions to sugarcane. The bacterial and fungal compositions at different taxonomic levels affected by clothianidin in the SPF and SP (with pesticide addition) were different from the effects experienced in the other treatments. With organic fertilizers added to SPF, the abundance of soil beneficial bacteria Bacillus, Paenibacillus, and Brevibacillus were highly improved, as well as the microbial function. It indicated that the SPF treatment could counteract the effects caused by mixing with clothianidin when compared to the SP treatment with only pesticide addition. Moreover, four bacterial genera including Dyella, Sphingomonas, Catenulispora, Mucilaginibacter, and Tumebacillus were significantly increased in the SPF and SP groups, which was reported to degrade clothianidin and could improve the soil health. The findings of the study provide insights into the interaction between the rhizosphere soil microbiome and a pesticide-fertilizer integration that may help improve application for pesticide-fertilizer in sugarcane fields.
\end{abstract}

\section{Introduction}

Sugarcane has become the most important cash crop in southern China. During the lifetime of sugarcane crop, it suffers from various pests in different development stages, and requires a large amount of pesticides and fertilizers to sustain its growth and health (Franco et al., 2011; Cherry et al., 2017). However, the heavy use of pesticides and fertilizers in sugarcane cultivation causes serious impacts on the agricultural environment, including agricultural non-point source pollution, increasing cost efficiency, energy conservation and emission, and effects on beneficial invertebrates, as well as threats to food safety and quality (Bokhtiar \& Sakurai 2005; Klaine et al., 2010; Velasco et al., 2012). In 2015, China's Ministry of Agriculture introduced two actions that seek to achieve zero growth in the use of chemical fertilizers and pesticides by 2020 (Jin \& Zhou, 2018).

Pesticide-fertilizer combinations or mixture products have been thriving and are increasingly applied in the farmland, as they serve a dual purpose with both nutritional and insecticidal characteristics, which could help achieve sugarcane pesticide-fertilizer integration and save labor and costs (Achorn \& Wright, 1982; Xie et al., $2017 \&$ 2018). Tiange, for instance, is a popular multifunctional pesticide-fertilizer product used in sugarcane cultivation in southern China, could effectively suppress pests including sugarcane borer, thrip, aphid, and cockchafer (Xie et al., 2017). Xie et al. (2019) found that the combination of 50\% organic fertilizer replacement and $50 \%$ pesticide reduction can produce the optimal results for both mitigating nutrient loss 
and balancing tea yield and quality. Yein et al. (2013) reported that pesticides and fertilizers in combination were much more effective at controlling the root-knot nematode infesting mung than using only pesticide or fertilizer alone. However, the domestic industry of pesticide and fertilizer integration is in its initial stage, the combination products currently lack industry standards and evaluation systems, and the potential impact on rhizosphere microbiome is still underestimated.

Rhizosphere microflora has been shown to be a key component of agricultural ecosystems that not only plays a significant role in basic soil processes but is also actively involved in enhancing soil fertility and crop productivity (Haney et al., 2015; Huang et al., 2020; Korenblum et al., 2020). However, research on the effects of pesticide and fertilizer combinations (SPF) on the rhizosphere microbial diversity and function of sugarcane is still rare. Currently, the overuse of synthetic fertilizers and pesticides has been reported to cause adverse effects on the soil microflora, and can substantially influence plant health and productivity (Hartmann et al., 2015; Zhu et al., 2016). In particular, fertilizers and pesticides tend to have long persistence in the soil, which is bound to affect the soil microflora and consequently disturbing soil health (Prashar \& Shah, 2016). Previous studies have found that chemical and bio-organic fertilizers have distinctive effects on the structure and function of the soil microbiome (Cai et al., 2017; Xiong et al., 2017). Compared with organic fertilizers, chemical fertilizers have many disadvantages, despite the ability to stimulate the growth of the fast-growing microbes by introducing more readily-available nutrients (Esperschütz et al., 2007). For instance, a high concentration of nitrogen fertilizer strongly modifies the composition of the soil microbiome, which could negatively impact soil carbon cycling and promote the abundance of fungal genera (PaungfooLonhienne et al., 2015). On the contrary, bio-fertilizers can improve soil health by direct suppression of pathogens or via modification of the indigenous microbial community (Xiong et al., 2017). In addition, organic fertilizers introduce more organic carbon into the soil and hold a closer neutral $\mathrm{pH}$ value, which is considered a dominant factor in determining the composition of soil microbiomes (Pang et al., 2017).

Investigating shifts driven by SPF in the composition of the soil microbiome would be helpful for maintaining and/or enhancing soil fertility and productivity, as well as for protecting soil ecosystems against disturbances (Tian et al., 2015). In this study, we hypothesized that pesticide inputs supplemented with organic fertilizer could maintain the composition of the soil microbiome more sustainably than the conventional method based on applying the pesticide and fertilizer separately. Here we conducted a field experiment to reveal any shifts in the rhizosphere microbiome with different pesticide and/or fertilizer treatments, as well as to compare treatment effects on the structure and function of the soil microbiome. The objectives of this study were to: (1) explore the effects of a SPF (pesticide and fertilizer combinations) on the structure and diversity of sugarcane's rhizosphere microbiome; and (2) reveal the potential interactions between the dual reductions in pesticide and fertilizer use and sugarcane quality.

\section{Materials and Methods}

\subsection{Experimental design}

SP1308 product was the SPF used in this study, which is applied widely in a sugarcane field in Guangdong Province. SP1308 contains two main components: clothianidin, which is a pesticide, and an animal-derived amino acid solution used as a bio-fertilizer. SP1308 contains clothianidin (1\%) (AccuStandard Company, USA), organic fertilizer (amino acid solution mainly including animal hair protein, $64 \mathrm{~mL} / \mathrm{kg}$ ), and excipients (aminocellulose $30 \mathrm{~g} / \mathrm{kg}$, gelatin $20 \mathrm{~g} / \mathrm{kg}$, and kaolin $940 \mathrm{~g} / \mathrm{kg}$, all produced by Damao company, Tianjin, China). Clothianidin ((E)-1-(2-chloro-1,3-thiazol-5-ylmethyl)-3-methyl-2-nitroguanidine), is a second-generation neonicotinoid insecticide; it is a white crystalline compound with the molecular weight of 249.68 and has the lowest water solubility $(0.327 \mathrm{~g} / \mathrm{l})$ among the neonicotinoids (Hirano et al., 2015). Clothianidin was determined to be a more persistent insecticide than imidacloprid and thiamethoxam in the soil of tropical sugarcane agroecosystems (Ramasubramanian et al., 2013). It was reported to have excellent plant systemicity, broad-spectrum activity, and confer high crop safety (Uneme \& Hideki, 2011). In addition to the insecticide effects, it has beneficial effects on the initial growth of sugarcane, increasing the total weight of plants and plant height, improving the root system, and even minimizing the effects of drought stress on the sugarcane (Endres et al., 2016). 
The experimental design was comprised of four groups, including (1) SPF (pesticide and fertilizer mixed with excipient), (2) a pesticide control (SP, pesticide mixed with excipient, but with no fertilizer added), (3) a fertilizer control (SF, fertilizer mixed with excipient, but with no pesticide added), and (4) an excipient control (SA, with neither pesticide nor fertilizer added), and one control blank (SK), with four replicates of each group.

\subsection{Field experiment, sampling and DNA extraction}

The field experiment was performed in a plot system located in Suixi County, Zhanjiang City of Guangdong Province $\left(21^{\circ} 14^{\prime} 23^{\prime \prime} \mathrm{N}, 110 \operatorname{deg} 4{ }^{\prime} 28^{\prime \prime} \mathrm{E}\right)$ to evaluate the effect of clothianidin with/without fertilizer on the structure of microbial communities in the rhizosphere and in bulk soil. When the sugarcane seedlings grew with $3 \sim 5$ leaves, we stated the field experiment including four treatments SPF, SP, SF, and SA with $100 \mathrm{~g}$ of each spreading into the soil close to sugarcane per plot $(7.4 \times 1.8 \mathrm{~m}$ in size), and a thin layer of soil applied to cover. Each treatment consisted of 4 plots and one meter between each plot, as well as a blank control.

After four weeks, rhizosphere soil and bulk samples were collected from sugarcane plots. For the rhizosphere samples, the complete root systems of $3^{\sim} 5$ plants per plot were combined as a composite sample after removing loosely adhering soil by vigorous shaking. 20 composite samples deposited in sterile zip-lock bags were carried to the lab with ice. Roots with soil on the surface were washed with a sterile 1 x PBS (phosphate-buffered saline) solution (Solarbio Life Sciences, China), and then centrifuged at 8,000 x g for $10 \mathrm{~min}$ at $4 \mathrm{degC}$. The resulting pellet, defined as the sample containing the rhizosphere-enriched microbial communities, was frozen in liquid nitrogen and stored at -80degC.

DNA extraction was performed using a soil DNA isolation kit (Tiangen Biotech, Beijing, China). DNA concentration and purification were quantified by Nanodrop 2000 as well as checked on $1 \%$ agarose gels for quality. The average DNA yield was 2-5 $\mu \mathrm{g}$ DNA with the concentration of $10-50 \mathrm{ng} / \mu \mathrm{L}$. DNA concentrations were additionally estimated using a Qubit 2.0 fluorometer (Thermo Fisher Scientific, USA).

\subsection{High-throughput amplicon sequencing}

Amplicon sequencing of the bacterial and fungal rhizosphere populations for 20 soil samples was conducted with the V4 fragment of the 16S rRNA gene and the ITS1 gene fragment, respectively, using the Illumina HiSeq platform (Illumina, San Diego, CA, USA). The broadly conserved primers, 515F, 5'GTGCCAGCMGCCGCGG-3', and 806R, 5'-GGACTACHVGGGTWTCTAAT-3', were used for PCR to amplify the sequencing region of the $16 \mathrm{~S}$ rRNA gene. PCR primers for amplifying fungal ITS1-2 are: ITS11737F, 5'-GGAAGTAAAAGTCGTAACAAGG-3'; ITS2-2043R, 5'-GCTGCGTTCTTCATCGATG C-3'.

PCR reactions were carried out in $30 \mu \mathrm{L}$ reactions with $15 \mu \mathrm{L}$ of Phusion@ High-Fidelity PCR Master Mix (New England Biolabs, USA), $0.2 \mu \mathrm{M}$ of forward and reverse primers, and about $10 \mathrm{ng}$ of template DNA. Thermal cycling consisted of initial denaturation at $98{ }^{\circ} \mathrm{C}$ for $1 \mathrm{~min}$, followed by 30 cycles of denaturation at $98{ }^{\circ} \mathrm{C}$ for $10 \mathrm{~s}$, annealing at $50{ }^{\circ} \mathrm{C}$ for $30 \mathrm{~s}$, elongation at $72{ }^{\circ} \mathrm{C}$ for $30 \mathrm{~s}$, and finally $72{ }^{\circ} \mathrm{C}$ for $5 \mathrm{~min}$. The same PCR conditions were used for ITS, except that the second stage had 35 cycles. PCR products were subsequently subjected to electrophoresis on a $2 \%$ agarose gel, stained with ethidium bromide, and the targeted fragment size purified with an AxyPrepDNA gel extraction kit (Axygen, China). Sequencing libraries were generated using Ion Plus Fragment Library Kit 48 rxns (Thermo Scientific, USA), following the manufacturer's recommendations. The library quality was assessed on the QuantiFluorTM-ST fluorometer (Promega) and Agilent 2100 Bioanalyzer system (Agilent, USA). Lastly, the library was sequenced on an Ion S5TM XL platform, and $400 \mathrm{bp} / 600 \mathrm{bp}$ single-end reads were generated. All the high-throughput sequencing was done by Novogene Technology Co, Ltd. (Beijing, China).

\subsection{Bioinformatics and Statistical analysis}

The raw sequences were processed using QIIME (version 1.9.1) (Caporaso et al., 2011). Adaptors and primers were removed using AdapterRemoval (Lindgreen, 2012). Phix contamination was removed using the DeconSeq program (Schmieder \& Edwards, 2011). Reads were merged and filtered by size (according to primer set) and quality (Phred quality score $>2$ ). The sequences were then clustered into operational taxonomic units 
(OTUs) using an open reference strategy based on a 97\% identity with the GreenGenes Database (v13_5 (DeSantis et al., 2006) as the reference. Taxonomy was assigned with an RDP classifier (Wang et al., 2007) retrained with SILVA (Release 115 http://www.arb-silva.de) for bacterial 16S rRNA gene database, as well as with UNITE (v7.2) (https://unite.ut.ee/) for fungal ITS database. OTUs assigned to chloroplasts and mitochondria were filtered out from the data set. Chimeric OTUs were identified using uchime (version 4.2 http://drive5.com/usearch/ manual/ uchime_algo.html) and removed from the OTU table.

All statistical analyses were carried out in R. Chao and Ace ((http://www.mothur.org) were calculated to characterize the community richness; Shannon index and Simpson index were calculated to characterize the community diversity. Rarefaction curves, reflecting the sequencing depth, were calculated using custom $\mathrm{R}$ scripts. To characterize the richness in a specific rhizosphere community, the custom $\mathrm{R}$ scripts were used to obtain Shannon-Wiener curve, Venn diagrams, and the microbial community bar plots. In the $\beta$-diversity analyses, $R$ package vegan was used to obtain the heat map. Principal coordinate analyses (PCoA) utilizing the weighted UniFrac distances were calculated using the pcoa() function of the $\mathrm{R}$ package Ape (Paradis, Claude, \& Strimmer, 2004). Plots and figures were generated with R (version 3.2.1) using the vegan, plyr, beanplot, ggplot2, and vcd packages. Phylogenetic Investigation of Communities by Reconstruction of Unobserved States (PICRUSt) and FunGuild were used to predict the metabolic function of the microbial community including nitrogen, methane, and energy metabolisms (Nguyen et al., 2016). All data were analyzed using one-way ANOVA, being transformed when necessary. Post hoc tests were used to investigate relationships between main factors when interactions were not significant.

\section{Results}

\subsection{Bacterial and fungal richness and diversity}

For the entire sampling set, a total of 1,353,140 bacterial sequences (raw tags) with an average length of 253 $\mathrm{bp}$, and 1,150,116 fungal sequences with an average length of $240 \mathrm{bp}$, were identified. As a result of chimeral filtration and quality control, 1,279,641 bacterial and 1,101,677 fungal high-quality sequences (clean tags) were obtained (Appendix S1 \& S2). In addition, the total number of $16 \mathrm{~S}$ and ITS reads obtained from the 18 and 14 soil samples, respectively, with two and six samples not successfully sequenced of each. The phylogenetic relationship of more than 500 bacterial and 200 fungal genera that are identified in SPFtreated rhizosphere soil, respectively (Appendix Figure S1). Rarefaction curves of most samples tend to be flat, suggesting that reasonable sequencing depth has been attained, although extra rare bacterial taxa are likely present in the sample (Appendix Figure S2).

Shannon and Chao1, the community richness index, showed that the bacterial diversity of SPF-treated rhizosphere soil was significantly higher than that of SP-treated and bulk soil (SK) $(p<0.05$, Figure 1a,b). $\alpha$ diversity in the SF group was found significantly increased compared to that in SP group $(p<0.05)$. In addition, $\alpha$ diversity of the excipient treatment (SA) was also investigated and compared with the others, which showed distinctive increased in diversity compared with that of SP and SK $(p<0.05)$. It is likely that the excipients added into SPF, SP, and SF did not decrease the diversity of the rhizosphere bacterial community of sugarcane. Shannon and Chao1 of the fungal diversity in the SPF were the highest $(p<0.05$, Figure 1c,d), followed by SF and SP, and these indices were the lowest in the SA group, indicating that SPF has the best fungal community richness.

According to the principal coordinate analysis (PCoA) plots, the bacterial community structure of the PW treatment was separated from those of the other treated groups by PCo1 (49.88\%) and PCo2 (19.15\%) (Figure 2a). The PCoA analysis based on weighted UniFrac metrics placed the bacterial community of SPF-treated soil much closer to the SP- and SF- treated soils, while the SK treatment was distantly placed. Beta-diversity between five groups also demonstrated that there were significant differences on bacterial community structure between SPF and SP, between SP and SA, between SP and SK, separately $(p<0.05$, Wilcox test, Appendix Figure S3a). In addition, Adonis (permutational MANOVA) showed that there were significant differences on bacterial communities between SP and SF ( $22=6.87, p<0.05)$. The fungal communities of each group were separated by PCo1 (78.44\%) and PCo2 (5.52\%) based on weighted UniFrac 
analysis (Figure $2 \mathrm{~b}$ ). The fungal community structure of SPF was much similar to that of SP, but significantly distinct from SF $(p<0.05$, Appendix Figure S3b).

\subsection{Microbial Community Composition and Structure}

For rhizosphere bacterial community in different treatments, the dominant bacterial phyla ( $>4 \%$ in any sample) were Proteobacteria, Actinobacteria, Firmicutes, Acidobacteria, and Bacteroidetes (Figure 3a). These phyla were represented in all groups with different relative abundances. Proteobacteria and Actinobacteria had the highest number of reads in each rhizosphere, together making up at least $60 \%$ of the total bacteria population in each set of samples. The main effects of pesticide and fertilizer addition on the bacterial community composition of the sugarcane rhizosphere and the interaction between both were studied using PERMANOVA analysis. The addition of pesticide (SP) or fertilizer (SF) had a significant effect on the bacterial community $(p<0.05)$, and there was an interaction effect on SPF with almost equivalent abundance between Proteobacteria (34.7\%) and Actinobacteria (30.9\%). While, Actinobacteria showed significantly increased in SP $(43.0 \%)$ compared with that in SF $(16.5 \%)(p<0.01)$, and Proteobacteria was significantly increased in SF $(48.1 \%)$ compared with that in SP $(23.2 \%)(p<0.05)$ (Appendix Figure S4a).

At the family level, the bacterial communities in all groups were dominated by Burkholderiaceae, Moraxellaceae, Lactobacillaceae, Streptomycetaceae, Micrococcaceae, Bacillaceae, and Enterobacteriaceae (Figure $3 \mathrm{~b}$ ). The relative abundance of many families varied greatly among different groups, especially families affected by the SP and SF groups, respectively. The abundance of Micrococcaceae and Streptomycetaceae in SP were significantly increased compared with those in SF $(p<0.01)$, while Moraxellaceae and Bacillaceae showed significantly increased in SF compared with those in SP $(p<0.01)$ (Appendix Figure S4b). Variation in the family abundance of SPF was more likely affected by both SP and SF addition.

In the top 20 genera, the dominant genera had distinct differences among different treatments. The abundance of Dyella ,Chryseobacterium, Lysinibacillus, and Acidothermusin SPF showed significant higher compared with those in other groups $(p<0.05$, Figure 3c). The abundance of Conexibacter, Leifsonia ,Chujaibacter, Sinomonas, and Acidipia in the SP treatment was significantly higher than that of the SA (excipients addition) and SK (blank control) $(p<0.05)$. It indicated that the distinctive genera in the group of pesticide addition (SP) could be relevant to the different processes of degeneration and metabolism of pesticides.

The dominant fungal phylum across all the groups was Ascomycota, followed by Basidiomycota and $\mathrm{Mu}-$ coromycota (Figure 4a). Among the top ten phyla, Mortierellomycota, Chytridiomycota, Glomeromycota, Blastocladiomycota, Rozellomycota, Aphelidiomycota, and Kickxellomycota were minor phyla, with an average relative abundance of less than $1 \%$. As shown in Figure $4 \mathrm{~b}$, the relative abundance of the fungal families diverged significantly among different groups. Specifically, the relative abundance of Chaetomiaceae was greatly increased in SP compared with that in SF $(p<0.01$, Appendix Figure S4c). The relative abundance of the top 10 families were much closer between SPF and SP, compared with other groups. In the top 20 genera, the bacterial communities after the SPF treatment were dominated by Penicillium(10.4\%), Talaromyces (10.3\%), followed by Trichoderma(4.7\%) and Myceliophthora (4.4\%) (Figure 4c). Compared with other groups using ANOVA analysis, the abundance of Nigrospora ,Deconica, Fusarium, Neocosmospora , Dokmaia ,Pachykytospora, and Ceratobasidium showed significantly increased in SPF $(p<0.05)$. The abundance ofMycelliophthora, Corynascus, and Mortierella both in the SPF and SP groups were much higher compared with those in the SF group $(p<0.05)$.

\subsection{Functional potential of the rhizosphere microbial community}

Bacterial functional profiles were inferred from 16S rRNA gene data (Appendix S3) using PICRUSt. Differences between the control and test groups were observed with a strong segregation $(p<0.05)$, including the notable enrichment of numerous pathways including peptidases, amino acid metabolism, starch and sucrose metabolism, porphyrin and chlorophyll metabolism, carbon fixation, flagellar assembly, and glycosyltransferases (Figure 5a, Appendix S4). Biological functions were found significantly abundant in the SPF treatment compared with those in the SK treatment $(p<0.05)$, including lipid metabolism, cell motility, 
metabolism of amino acids, glycan biosynthesis, and xenobiotics biodegradation. Through comparisons of the bacterial function in groups SPF and SP using t-test, we found that SPF exhibited significantly higher xenobiotics biodegradation and metabolism $(p<0.05)$ (Appendix Figure S5a). SPF had significantly increased functions for metabolism of cofactors and vitamins compared to SF $(p<0.05)$ (Appendix Figure $\mathrm{S} 5 \mathrm{~b})$, as well as increased functions of metabolism compared to SA $(p<0.05)$ (Appendix Figure S5c). As the only different compound between the SP and SF groups was clothianidin, the distinctive functions caused by clothianidin mainly included pyruvate metabolism, glycolysis, carbon fixation, butanoate metabolism, and methane metabolism, which were significantly higher than that in the SF treatment $(p<0.05)$ (Figure $5 a)$. This results demonstrates that these functions would be positively involved in the transformation and degradation of clothianidin in soil.

We allocated 1494 total fungal OTUs (Appendix S5) to fungal functional groups using the FUNGuild annotation tool. At least eight trophic modes were detected in this study, among which the saprotroph mode was the most abundant, followed by pathotroph, pathotroph-saprotroph, pathotroph-saprotroph-symbiotroph, and symbiotroph (Figure 5b, Appendix S6). Sequences annotated with the saprotroph, pathotroph-saprotroph and pathotroph-symbiotroph modes were significantly decreased in the SPF group $(p<0.05)$ than those in the SK and SA groups. At the guild level, the most abundant guilds were saprotrophs, followed by plant pathogens, soil saprotrophs, and wood saprotrophs (Figure 5c, Appendix S7). The relative abundances of different fungal functional guilds also differed significantly between groups. Specifically, SPF had a significantly higher abundance of soil saprotroph and wood saprotroph compared to other groups $(p<0.05)$. However, SA and SK had significantly higher abundances of plant pathogen $(p<0.05)$ compared to that in SPF (Figure 5c).

The abundance of functional genes associated with nitrogen cycling, including nifB , nifH , nifU, and nirZ , in each 16S library was analyzed (Appendix Figure S6, Appendix S8). The relative abundances of these four genes of the SPF treatment showed no significant difference compared to other treatments, indicating that the nitrification metabolism in soil was not affected by pesticides, fertilizers, and/or excipients addition. We also found that the SF group with organic fertilizer addition, it significantly improved the abundance of soil beneficial bacteria Bacillus andPaenibacillus compared to the SA and SK groups without fertilizer addition $(p<0.05)$ (Figure 6). Brevibacilluswas also highly enriched in the SF group compared with that in the SA, SK, and SP groups $(p<0.01)$. The abundances of these three bacterial genera in SF also showed higher than other groups except for the SPF, which demonstrated that the SPF group with the organic fertilizer addition would be low harm to the soil environment when applied to the sugarcane field.

\section{Discussion}

To estimate agrochemical compounds including pesticide and fertilizer mixtures is one of the most important factors in evaluating the toxicological or ecological impacts of these compounds (Schmitz et al., 2014). In our study, SPF is an agrochemical compound widely used on sugarcane seedlings in South China, which principal components including pesticide (clothianidin, a chemical compound) and an organic fertilizer. However, there is less researches about its impact on soil and water as well as relevant insects and microbes. This study was conducted to investigate the effects of SPF on the rhizosphere microbial community of fieldgrown sugarcane. Here we conducted $16 \mathrm{~S}$ rRNA gene amplicon sequencing to detect the disturbance to the diversity and structure of rhizosphere microbiota under different pesticide and/or fertilizer treatments. We found that the bacterial and fungal compositions at the phylum level affected by clothianidin in the SPF and SP groups were different from the effects experienced in the other treatment groups. Meanwhile, the structure of Proteobacteria, Actinobacteria, Firmicutes, Acidobacteria, and Actinobacteria in these different groups was variable with different relative abundances (Figure 3a). While, the abundances of fungal phyla including Ascomycota and Basidiomycota in the SPF and SP groups were almost the same, indicating that the dominant fungi would be more affected by clothianidin (Figure 4a). As expected, the application of a chemical compound clothianidin was a major factor shaping the rhizosphere microbial community taxonomic structure (Zhu et al., 2016; Diez et al., 2017). However, organic fertilizers addition was proved to be able to enrich the microbial diversity (Cai et al., 2017) and could counteract the effects caused by mixing with 
clothianidin in the SPF treatment soil when compared with the SP group with only pesticide addition. The effects of SPF with organic fertilizer addition on the rhizosphere microbiome were also found not to be significant as revealed by the diversity (Figure 1) and function (Figure 5) as the microbial community of SPF compared to the SP group.

As is reported, it usually involves more than one microorganism when pesticide degradation occurs in soil (Doolotkeldieva et al., 2018). Comparing to the SK control treatment, the more abundant phyla present in the SPF treatment, such as Bacteroidetes, Firmicutes, and Acidobacteria, have been reported to be pesticidetolerant bacteria and could be responsible for the biodegradation of applied pesticides (Hassen et al., 2018). The bacterial families Burkholderiaceae and Streptomycetaceae with enriched abundances shown in the SPF and SP groups (Figure 3b) are considered to be active microbiodegraders (Mamta \& Khursheed, 2015). Four bacterial genera including Dyella ,Sphingomonas, Catenulispora, Mucilaginibacter, and Tumebacillus were found highly increased in the SPF and SP groups, which could be able to degrade clothianidin (Polivkova et al., 2018). In particular, Dyella ginsengisoli was found to be a highly efficient biphenyl-degrading strain that could accelerate the startup period of the biphenyl bioremediation process (Zhao et al., 2010). If these clothianidin degrading genera found in this study could be isolated and cultured, it would be helpful to improve soil quality with external addition to clothianidin-contaminated farmlands. In addition, some strains in Ascomycota of this study, the dominant fungal phylum in all groups, have been reported to produce laccase with high yield, and thus have potential applications in environmental protection, e.g., decoloring the wastewater (Neoh et al., 2014), as well as biomass conversion (Xie et al., 2014).

The alpha-diversity of the bacterial communities in the SA treatment compared with the SPF and SF treatments were found not significantly different but showed highly increased compared to SP (Figure 1). This suggested that the common excipients added to the SPF would not cause the significant disturbance of diversity in the soil microbial community. For the functional prediction of the bacteria community, the SPF treatment also showed more positive biological metabolism functions, including pyruvate metabolism, glycolysis, carbon fixation, butanoate metabolism, methane metabolism, and pantothenate biosynthesis, which indicated it would not have severe negative impacts on the soil environment. The functional comparison was also conducted between the excipient treatment (SA) and the SK control treatment, showing lower adverse effects on the rhizosphere bacteria community of sugarcane. On the contrary, the function of the SA-treated bacterial community showed more significantly enhanced $(p<0.05)$ in their biochemical cycling, including oxidative phosphorylation, nitrogen metabolism, sulfur metabolism, and glutathione metabolism, as compared to the SK treatment (Figure 5a). This indicated the excipient mixture we used in this study (added into the SPF, SP, and SF treatments) was not too harsh on the bacterial diversity and function of soil. The fungal functions revealed by FunGuild.mode also showed that there was much lower proportion of the SPF-treated soil to become pathotroph type compared to SA and SK (Figure 5b). In addition, clothianidin used in this study was reported to be a decomposable pesticide taking about 20-25 days to decompose in the soil (Yang et al., 2018), which would cause less stress on the soil environment. Moreover, the SPF treatment with the organic fertilizer addition applied to filed-grown sugarcane could release the strong rhizosphere effects caused by clothianidin, which would improve the root biomass and health of sugarcane seedlings.

In this study, we investigated the effects of a pesticide-fertilizer combination, as well as the mixture of single active compounds, on the sugarcane rhizosphere soil microflora in an attempt to understand the possible interaction effects. The results, based on field experiments, revealed that using a pesticide-fertilizer mixture in the field-grown sugarcane does not adversely affect the diversity and function of soil microbiome. Moreover, appropriate organic fertilizer input and pesticide reduction management practices are not only helpful for decreasing the labor cost of sugarcane cultivation, but also beneficial to reducing the potential risk of environmental pollution. Therefore, the interactive effects of different fertilizer and pesticide managements on the rhizosphere microbiome will be extremely helpful in determining a reasonable application scheme for the fertilizers and pesticides.

\section{Acknowledgments}

This work was funded by GDAS' Project of Science and Technology Development (2019GDASYL-0103031), 
and National Key R\&D Program of China (2018YFD0201100).

\section{Competing interests}

The authors declare that they have no competing interests.

Author Contributions

W.H. and Y.A. designed the work; Y.L. and L.C. performed the field collections; W.H. performed the laboratory research and analyzed the data; W.H. and Y.A. wrote the manuscript.

\section{Data Accessibility}

The metagenome sequences of all samples were uploaded to NCBI SRA database with the accession number SUB7134467 and SUB7134514.

Supporting Information: 1 Appendix Figures file; 1 Appendix Datasets file.

\section{References}

Achorn, F.P., Wright, E.B. (1982). Production of fertilizer-pesticide mixtures. ASTM special technical publication, USA, 764, 18.

Bokhtiar, S.M., Sakurai, K. (2005). Effect of application of inorganic and organic fertilizers on growth, yield and quality of sugarcane.Sugar Tech , 7(1), 33-37.

Cai, F., Pang, G., Li, R.X., Li, R., Gu, X.L., Shen, Q.R., Chen, W. (2017). Bioorganic fertilizer maintains a more stable soil microbiome than chemical fertilizer for monocropping. Biology and Fertility of Soils , 53(8), 861-872.

Caporaso, J.G., Lauber, C.L., Walters, W.A., Berg-Lyons, D., Lozupone, C.A., Turnbaugh, P.J., Fierer, N., Knight, R. (2011). Global patterns of 16S rRNA diversity at a depth of millions of sequences per sample.Proceedings of the National Academy of Sciences of the United States of America, 108, Suppl 1, 4516-4522.

Cherry, R., Mccray, M., Sandhu, H. (2017). Changes in the relative abundance of soil-dwelling insect pests in sugarcane grown in Florida. Journal of Entomological Science, 52(2), 169-176.

DeSantis, T.Z., Hugenholtz, P., Keller, K., Brodie, E.L., Larsen, N., Piceno, Y.M., Phan, R., Andersen, G.L. (2006). NAST: a multiple sequence alignment server for comparative analysis of $16 \mathrm{~S}$ rRNA genes. Nucleic Acids Research, 34, Suppl 2, W394-W399.

Diez, M.C., Elgueta, S., Rubilar, O., Tortella, G.R., Schalchli, H., Bornhardt, C., Gallardo, F. (2017). Pesticide dissipation and microbial community changes in a biopurification system: influence of the rhizosphere. Biodegradation, 28(5-6), 1-18.

Doolotkeldieva, T., Konurbaeva, M., Bobusheva, S. (2018). Microbial communities in pesticide-contaminated soils in Kyrgyzstan and bioremediation possibilities. Environmental Science and Pollution Research , 25, 31848-31862.

Edwards, J., Johnson, C., Santos-Medellin, C., Lurie, E., Podishetty, N.K., Bhatnagar, S., Eisen, J.A., Sundaresan, V. (2015). Structure, variation, and assembly of the root-associated microbiomes of rice.Proceedings of the National Academy of Sciences of the United States of America, 112(8), E911.

Endres, L., Oliveira, N.G., Ferreira, V.M., Silva, J.V., Barbosa, G.V., Sebastião, O.M. (2016). Morphological and physiological response of sugarcane under abiotic stress to neonicotinoid insecticides. Theoretical and Experimental Plant Physiology, 28(4), 1-9.

Franco, H.C.J., Otto, R., Faroni, C.E., André, C.V., Emídio, C.A.O., Trivelin, P.C.O. (2011). Nitrogen in sugarcane derived from fertilizer under Brazilian field conditions. Field Crops Research , 121(1), 29-41. 
Hartmann, M., Frey, B., Mayer, J., Mäder, P., Widmer, F. (2015). Distinct soil microbial diversity under long-term organic and conventional farming. The ISME Journal , 9(5), 1177.

Haney, C.H., Samuel, B.S., Bush, J., et al. (2015). Associations with rhizosphere bacteria can confer an adaptive advantage to plants. Nature Plants , 1(6):15051.

Hassen, W., Neifar, M., Cherif, H., Mahjoubi, M., Souissi, Y., Raddadi, N., Fava, F., Cherif, A. (2018). Assessment of genetic diversity and bioremediation potential of pseudomonads isolated from pesticide-contaminated artichoke farm soils. 3 Biotech, 8(6), 263.

Hirano, T., Yanai, S., Omotejara, T., Hashimoto, R., Umemura, Y., Kubota, N., ... Hoshi, N. (2015). The combined effect of clothianidin and environmental stress on the behavioral and reproductive function in male mice. Journal of Veterinary Medical Science , 77(10), 1207-1215.

Huang WJ, Sun DL, Fu JT, et al. (2020). Effects of continuous sugar beet cropping on rhizospheric microbial communities. Genes, 11, 13.

Jin, S.Q., Zhou, F. (2018). Zero growth of chemical fertilizer and pesticide use: China's objectives, progress and challenges. Journal of Research Ecology, 9(1), 50-58.

Klaine, S.J., Hinman, M.L., Winkelmann, D.A., Sauser, K.R., Martin, J.R., Moore, L.W. (2010). Characterization of agricultural nonpoint pollution: Pesticide migration in a West Tennessee watershed.Environmental Toxicology and Chemistry, 7(8), 609-614.

Korenblum E, Dong YH, Szymanski J, et al. (2020). Rhizosphere microbiome mediates systemic root metabolite exudation by root-to-root signaling.Proceedings of the National Academy of Sciences of the United States of America, 117 (7), 3874-3883.

Lindgreen, S. (2012). AdapterRemoval: easy cleaning of next-generation sequencing reads. BMC Research Notes , 5(1), 337.

Mamta, R.J.R., Khursheed, A.W. (2015). Bioremediation of pesticides under the influence of bacteria and fungi Chapter 3, Source Title: Handbook of Research on Uncovering New Methods for Ecosystem Management through Bioremediation, pp 51-72.

Neoh, C. H., Lam, C. Y., Lim, C. K., Yahya, A. \& Ibrahim, Z. (2014). Decolorization of palm oil mill effluent using growing cultures of Curvularia clavata . Environmental Science and Pollution Research , 21, 4397-408.

Nguyen, N.H., Song, Z., Bates, S.T., Branco, S., Tedersoo, L., Menke, J., Schiling, J.S., Kennedy, P.G. (2016). FUNGuild: An open annotation tool for parsing fungal community datasets by ecological guild.Fungal Ecology , 20, 241-248.

Ramasubramanian, T. (2013). Persistence and dissipation kinetics of clothianidin in the soil of tropical sugarcane ecosystem. Water Air and Soil Pollution, 224(3), 1-5.

Schmieder, R., Edwards, R. (2012). Fast identification and removal of sequence contamination from genomic and metagenomic datasets. PLoS ONE , 6(3), e17288.

Schmitz, J., Hahn, M., Brühl, C.A. (2014). Agrochemicals in field margins - An experimental field study to assess the impacts of pesticides and fertilizers on a natural plant community.Agriculture Ecosystems and Environment , 193, 60-69.

Pang, G., Cai, F., Li, R.X., Zhao, Z., Li, R., Gu, X.L., Shen, Q.R., Chen, W. (2017). Trichoderma -enriched organic fertilizer can mitigate microbiome degeneration of monocropped soil to maintain better plant growth. Plant and Soil , 416(1-2), 1-12.

Paradis, E., Claude, J. \& Strimmer, K. (2004). APE: analyses of phylogenetics and evolution in R language. Bioinformatics, 20, 289-290. 
Polivkova, M., Suman, J., Strejcek, M., Kracmarova, M., Hradilova, M., Filipova, A., Cajthaml, T., Macke, T., Uhlik, O. (2018). Diversity of root-associated microbial populations of Tamarix parvifloracultivated under various conditions. Applied Soil Ecology , 125, 264-272.

Prashar, P., Shah, S. (2016). Impact of Fertilizers and Pesticides on Soil Microflora in Agriculture. In: Lichtfouse E. (eds) Sustainable Agriculture Reviews. Sustainable Agriculture Reviews, vol 19. Springer, Cham.

Uneme, H. (2011). Chemistry of clothianidin and related compounds. Journal of Agricultural and Food Chemistry , 59(7), 2932-2937.

Velasco, A., Rodríguez, J., Castillo, R., Ortíz, I. (2012). Residues of organochlorine and organophosphorus pesticides in sugarcane crop soils and river water. Journal of Environmental Science and Health , 47(9), 833-841.

Wang, Q., Garrity, G.M., Tiedje, J.M., Cole, J.R. (2007). Naive Bayesian classifier for rapid assignment of rRNA sequences into the new bacterial taxonomy. Applied and Environmental Microbiology , 73(16), 5261-5267.

Xie, J.J., Chen, Y.G., Peng, D.Y., Yang, C.Q., Huang, X.J., Wu, G., Chen, C., Yang, J.X. (2017). Effect of $30 \%$ Tiange multifunctional pesticide-fertilizer on sugarcane pests and yield. Asian Agricultural Research , $7,53-56$.

Xie, N., Chapeland-Leclerc, F., Silar, P. \& Ruprich-Robert, G. (2014). Systematic gene deletions evidences that laccases are involved in several stages of wood degradation in the filamentous fungusPodospora anserina . Environmental Microbiology , 16, 141-161.

Xie, S., Feng, H., Yang, F., Zhao, Z., Hu, X., Wei, C., Liang, T., Li, H.T., Geng, Y.B. (2019). Does dual reduction in chemical fertilizer and pesticides improve nutrient loss and tea yield and quality? A pilot study in a green tea garden in Shaoxing, Zhejiang Province, China.Environmental Science and Pollution Research , 26(3), 2464-2476.

Xiong, W., Guo, S., Jousset, A., Zhao, Q., Shen, Q. (2017). Bio-fertilizer application induces soil suppressiveness againstFusarium wilt disease by reshaping the soil microbiome.Soil Biology and Biochemistry , 114, 238-247.

Yang, Q., Liu, N., Cheng, G., Zhang, S., Zhou, Y., Liang, D., Gu, Z. (2018). Residue and dissipation of clothianidin in rice and soil.Agrochemicals , 5:343-346.

Yein, B.R., Singh, H., Chhabra, H.K. (2013). Effect of pesticides and fertilizers singly and in combination on the root-knot nematode infesting mung. Indian Journal of Nematology, 7(2), 117-122.

Zhao, L.J., Jia, Y.H., Zhou, J.T., Li, A., Chen, J.F. (2010). Dynamics of augmented soil system containing biphenyl with Dyella ginsengisoli LA-4. Journal of Hazardous Materials , 179(1-3), 729-734.

Zhu, S., Vivanco, J.M., Manter, D.K. (2016). Nitrogen fertilizer rate affects root exudation, the rhizosphere microbiome and nitrogen-use-efficiency of maize. Applied Soil Ecology , 107, 324-333.

\section{Hosted file}

Figures.pdf available at https://authorea.com/users/303248/articles/433370-impact-ofpesticide-fertilizer-combinations-on-the-rhizosphere-microbiome-of-field-grown-sugarcane 


\section{Figure Legends:}

Figure 1 Alpha-diversity of the bacterial and fungal communities for five groups of microbial samples.

a \& b, representing Shannon diversity and Chaol of the bacterial community; b \& d, representing Shannon diversity and Chaol of the fungal community. Significances between different groups were compared using Wilcoxon's test, with the results indicated on the top ( $p$-value $\leq 0.05=*, p$-value $\leq 0.01=* *$ ).

Figure 2 Beta diversity of the microbial community using PCoA analysis with the weighted_Unifrac metric. a, bacterial community; b, fungal community.

Figure 3 Taxonomic differences of the bacterial community among four groups of samples. a, the top 10 phyla;

b, the top 10 families; c, relative abundances of the top 20 genera were compared between different groups.

Figure 4 Taxonomic differences of the fungal community among four groups of samples.a, the top 10 phyla;

b, the top 10 families; c, relative abundances of the top 20 genera were compared between different groups.

Figure 5 Functional prediction of microbial community between five groups. a, bacterial community; b, annotation of fungal community using mode; $c$, annotation of fungal community using Guild.

Figure 6 Abundance of some representative soil beneficial bacteria ( $p$-value $\leq 0.05=*, p$-value $\leq 0.01=* *)$. 